Mol Inform. 2018 December ; 37(12): e1800053. doi:10.1002/minf.201800053.

\title{
Flap-site fragment restores back wild-type behaviour in resistant form of HIV protease
}

\author{
Adriano Luchi ${ }^{\mathrm{a}}$, Emilio Angelina ${ }^{*}, \mathrm{a}$, Lucrecia Bogado $^{\mathrm{a}}$, Stefano Forli ${ }^{\mathrm{b}}$, Arthur Olson ${ }^{\mathrm{b}}$, and \\ Nélida Peruchena ${ }^{\mathrm{a}}$ \\ [a]Lab. Estructura Molecular y Propiedades, IQUIBA-NEA, Universidad Nacional del Nordeste, \\ CONICET, FACENA. Av. Libertad 5470, Corrientes 3400, Argentina \\ [b]Molecular Graphics Lab, Department of Integrative Structural and Computational Biology, \\ MB-112, the Scripps Research Institute, 10550 North Torrey Pines Road, La Jolla, California \\ 92037-1000
}

\section{Abstract}

HIV-1 protease (HIV-PR) performs a vital step in the virus life cycle which makes it an excellent target for drug therapy. However, due to the error-prone of HIV reverse transcriptase, mutations in HIV-PR often occur, inducing drug-resistance to inhibitors. Some HIV-PR mutations can make the flaps of the enzyme more flexible thus increasing the flaps opening rate and inhibitor releasing. It has been shown that by targeting novel binding sites on HIV-PR with small molecules, it is possible to alter the equilibrium of flap conformational states. A previous fragment-based crystallographic screen have found two novel binding sites for small fragments in the inhibited, closed form of HIV-PR, termed flap and exo sites.

While these experiments were performed in wild type HIV-PR, it still remains to be proven whether these small fragments can stabilize the closed conformation of flaps in resistant forms of the enzyme.

Here we performed Molecular Dynamics simulations of wild type and mutant form of HIV-PR bound to inhibitor TL-3. Simulations show that on going from wild type to 6X mutant the equilibrium shifts from closed to semi-open conformation of flaps. However, when fragment Br6 is placed at flap site of mutant form, the enzyme is restored back to closed conformation. This finding supports the hypothesis that allosteric inhibitors, together with active site inhibitors could increase the number of point mutations necessary for appreciable clinical resistance to AIDS therapy.

\section{Keywords}

AIDS; allosteric inhibitor; Molecular Dynamics; Principal Component Analysis; QTAIM

*emilioluisangelina@hotmail.com, phone: (54)3794-457996 intern 101.

므 Supporting Information for this article is available on the WWW under www.molinf.com 


\section{Introduction}

HIV-1 protease (HIV-PR) performs a vital step in the life cycle of the Human immunodeficiency virus (HIV). HIV-PR break up gag / gag-pol polyproteins precursors into the mature protein components of an infectious virion. Drugs that are able to block HIV-PR function prevents virus maturation into its infective form. ${ }^{[1]}$

HIV-PR is a symmetrically assembled homo-dimer with the following structural segments: flap (residues 43-58/43'-58'), flap elbow (residues 35-42/35'-42'), fulcrum (residues 1122/11'-22'), cantilever (residues 59-75/59'-75'), dimerization interface (residues 15/1'-5', 95-99/95'-99'), active site (residues 23-30/23'-30'), 80s loop (76-84/76'-84') and helix (86-90/86'-90').

Ten protease inhibitors have been approved so far by the FDA, all of them bind to the active site of HIV-PR preventing virus maturation. ${ }^{[2]}$ Nevertheless, because of the high error-prone of HIV-1 reverse transcriptase (HIV-RT) mutations in HIV-PR often arise during the course of treatment, inducing drug-resistance to above mentioned active site inhibitors. ${ }^{[3]}$

Experiments and theoretical studies revealed that HIV-PR can be in any of three conformations of the flaps: closed, semi-open, and fully open, which coexists in a dynamic equilibrium. In the apo state flaps are mostly open so that substrate can enter the active site. After substrate binding the flaps close around it to start the cleavage. The equilibrium among the functional conformations is controlled by the flexibility of flap regions. Mutations in HIV-PR can make the flaps more flexible and destabilize the closed conformation, thus increasing flaps opening rate and inhibitor releasing. ${ }^{[2]}$

Available structural models of HIV-PR mutant forms offers the possibility to understand the mechanisms by which the enzyme can overcome inhibition. Heaslet et al. have obtained the crystal structures of three mutant forms of HIV-PR containing one (1X: V82A), three (3X: V82A, M46I, F53L) and six (6X: V82A, M46I, F53L, V77I, L24I, L63P) point mutations. The three protease mutants arise sequentially during tissue culture in the presence of increasing concentrations of potent inhibitor TL-3 and exhibit 4, 11 and 30-fold resistance to TL-3, respectively. ${ }^{[4]}$ The first mutation to arise in response to treatment with TL-3 was Val82 to Ala which is located in the 80s loop. This mutation perturbs hydrophobic interactions between flaps and 80s loop residues that help to maintain HIV-PR in the closed conformation. ${ }^{[2]}$ After V82A mutation, two additional mutations, Met46 to Ile and Phe53 to Leu, both localized in the flap region of the protease further increased the mobility of the flaps. The third step in the evolution toward resistance resulted in the $6 \mathrm{X}$ protease mutant. The new mutations in the $6 \mathrm{X}$ protease are all distal to the protease flap regions, but still they are able to substantially affect the TL-3 binding site. ${ }^{[4]}$

Various strategies have been applied to develop new antiretroviral therapies against drugresistant forms of HIV-PR, from increasing the plasma levels of existing protease inhibitors by using a boosting agent to developing new protease inhibitors using structure-based drug design. ${ }^{[5]}$ In a different approach Ung et al. has shown that by targeting novel binding sites on the surface of the HIV-PR with small molecules, it is possible to modulate the enzymatic 
activity of the protease through altering the dynamics of the flaps and the equilibrium of the flap conformational states. ${ }^{[6]}$

In line with this novel approach some of us have performed a fragment-based crystallographic screen against inhibitor-bound HIV-PR to search for compounds that bind to novel sites and stabilize the inhibited conformation of the target. ${ }^{[7]}$ The experiments have revealed two new binding sites for small fragments in the inhibited, closed form of the enzyme, termed flap site and exo sites; two of the 384 fragments screened bound in the flap site (1F1 and 1F1-N) and one in the exo site (4D9). Further work identified a third fragment hit that bound in the flap site and suggests that flap site binding favours a closed flap conformation of HIV-PR. ${ }^{[8]}$ More recently, Tiefenbrunn et al. have screened a 68-member brominated fragment library against HIV-PR and led to the identification of two new compounds that also bind to the flap site (Br6) and exo site (Br27). ${ }^{[9]}$ While these experiments were performed in wild type HIV-PR, it still remains to be proven whether these small fragments can stabilize the closed conformation of flaps in resistant forms of the enzyme.

In this work we have performed long Molecular Dynamics (MD) simulations of wild type (Wt) and 6X variant of HIV-PR bound to TL-3. The simulations show that when going from $\mathrm{Wt}$ to $6 \mathrm{X}$ the equilibrium shifts from closed to semi-open conformation of the flaps, in agreement with previous experimental and theoretical evidence. Then we placed small fragments $\mathrm{Br} 6$ and $\mathrm{Br} 27$ at flap site and exo site of 6X/TL-3 complex, respectively and performed MD simulations of the ternary complexes $6 \mathrm{X} / \mathrm{TL}-3 / \mathrm{Br} 6$ and $6 \mathrm{X} / \mathrm{TL}-3 / \mathrm{Br} 27$. To our surprise, one of the fragments, Br6, was able to restore back the enzyme to closed conformation of the flaps. That is to say, when Br6 is bound to one of the flaps of $6 \mathrm{X}$ it behaves much like the Wt form of HIV-PR. This finding supports the hypothesis already proposed by some of us ${ }^{[7]}$ that allosteric inhibitors when administered together with active site inhibitors could likely increase the number of protease mutations necessary for appreciable clinical resistance to the highly active anti-retroviral therapy (HAART).

\section{Computational Details}

\subsection{Simulation Protocol}

Initial coordinates of $\mathrm{Wt}$ and $6 \mathrm{X}$ variant bound to TL-3 were obtained from the RCSB Protein Data Bank (codes 2AZ8 and 2AZC, respectively). Starting configurations of small fragments Br27 and Br6 in ternary complexes 6X/TL-3/Br27 and 6X/TL-3/Br6 respectively, were manually constructed based in the $\mathrm{X}$-ray structures of $\mathrm{Br} 27$ bound to exo site (PDB code $4 \mathrm{~K} 4 \mathrm{R}$ ) and Br6 bound to the flap site (PDB code 4K4Q).

All the simulations were carried out in triplicate (i.e. replicas 1, 2 and 3) with Amber14 software package ${ }^{[10 \mathrm{a}]}$ at $300^{\circ} \mathrm{K}$ temperature and extended up to $0.4 \mu$ s overall simulation time in a truncated octahedral periodic box of TIP3P water molecules. Amber ff14SB force field was used for proteins. ${ }^{[10 \mathrm{~b}]}$ The antechamber software in the AmberTools package was used to generate ligands (i.e. TL3, Br6 and Br27) parameters with GAFF force field and AM1-BCC charges. ${ }^{[10 c]}$ Every replica was run with the same random seed for velocity 
profile generation, so that simulations within a replica are comparable among the different systems.

\subsection{Dynamic Cross Correlation Analysis}

Correlation between all proteins residues were analysed for the last $0.35 \mu$ s of each trajectory replica using the normalized covariance

$$
\mathrm{C}_{\mathrm{ij}}=\operatorname{Cov}_{i j} / \quad\left(\Delta \mathrm{r}_{\mathrm{i}}(\mathrm{t})^{2} \cdot \Delta \mathrm{r}_{\mathrm{j}}(\mathrm{t})^{2}\right)^{1 / 2}
$$

where $\operatorname{Cov}_{i j}=\left\langle\Delta \mathrm{r}_{\mathrm{i}}(\mathrm{t}) \cdot \Delta \mathrm{r}_{\mathrm{j}}(\mathrm{t})\right\rangle$ and $\Delta \mathrm{r}_{\mathrm{i}}(\mathrm{t})=\mathrm{r}_{\mathrm{i}}(\mathrm{t})-\left\langle\mathrm{r}_{\mathrm{i}}(\mathrm{t})\right\rangle$.

$r_{i}(t)$ is the position of node $i$ at time $t$ and $<>$ represent time averaging. In this study, the correlation coefficients $\mathrm{C}_{\mathrm{ij}}$ have been computed by considering backbone carbon atoms of protein residues. Correlations were represented graphically with the help of Bio3D R package. ${ }^{[11]}$

\subsection{Principal Components Analysis (PCA)}

PCA calculations were performed with python package ProDy ${ }^{[12]}$ on an ensemble of conformations derived from MD simulations to characterize collective protein motions. The resulting Principal Components (PCs) were sorted according to their contribution to the total fluctuation along the ensemble of conformations. It has been shown that only a small subset of these PCs describes a great majority of the total atomic displacement. ${ }^{[13]}$ The calculation of the PCs involves two main steps: (1) the calculation of the covariance matrix, $\operatorname{Cov}_{i j}$, of the positional deviations, and (2) the diagonalization of this matrix which is equivalent to solving the eigenvalue problem.

\subsection{Quantum Theory of Atoms in Molecules (QTAIM)}

MD trajectories were projected along the first PC (PC1) and frames with extreme values of projection were selected for subsequent charge density topological analysis using QTAIM. ${ }^{[14]}$ This analysis has been very useful for evaluating the interactions in bio-molecular systems. ${ }^{[15-18]}$

Since accurate quantum calculations are still forbidden for full bio-molecular complexes, reduced 3D models were constructed from minimum and maximum extreme projection structures of complexes Wt/TL-3, 6X/TL-3 and 6X/TL-3/Br6. Fifty-two residues were included in the models: residues I13, K14, I15, G16, G17, Q18 and K20 from fulcrum; T31, V32, L33, E34, E35, M36, N37, L38, P39 and W42 from flap elbow; P44, K45, M46 (I46 in $6 \mathrm{X}$ variant), I47, G48, G49, I50, G51, G52, F53 (L53 in 6X variant), I54, K55, V56, R57 and Q58 from flap; Y59, I62, L63 (P63 in 6X variant), I64, E65 from cantilever; V75, L76, V77 (I77 in 6X variant), G78, P79, T80, P81, V82 (A82 for the 6X mutated variant), N83, I84 and I85 from 80's loop. All these residues belong to monomer/chain A of the HIV-PR homo-dimmer while only four residues from monomer B flap tip were included in the reduced models: G49', I50', G51' and G52'. 
Once the reduced models were constructed the charge density was computed by DFT methodology with the M06-2x dispersion corrected hybrid functional and 6-31G (d) as basis set, as implemented in Gaussian 09 package. ${ }^{[19]}$ The topological analysis of charge density was then performed with the help of Multiwfn software. ${ }^{[20]}$

\section{Results and Discussion}

MD simulations of wild type and 6X mutant of HIV-PR were performed to investigate the effect of mutations on the dynamic of the flaps. Furthermore, simulations of $6 \mathrm{X}$ mutant bound to small fragments in the flap site and exo site were also carried out. In all system configurations the protease was bound to the same active site inhibitor TL-3.

\subsection{Effect of mutations on the flexibility of the flaps}

To inspect the motion of flaps along the MD trajectories, the distance I50-I50' between residues from the tip of the flaps is depicted in Figure 1 for different system configurations.

Comparison of $\mathrm{Wt} / \mathrm{TL}-3$ and 6X/TL-3 complexes clearly shows that mutations in the protease turn flaps more prone for opening (see Figure 1). This finding is in agreement with previous studies that have shown that mutations in HIV-PR make the flaps more flexible and destabilize closed conformation. ${ }^{[2,4]}$

To investigate the effect of non-active site binders on the flexibility of $6 \mathrm{X}$ mutant flaps, MD simulations of this protease variant with small fragment 1-bromo-2-naphthoic acid (Br27) bound to exo site and 3-bromo-2,6-dimethoxybenzoic acid ( $\mathrm{Br} 6)$ bound to flap site were performed.

Previous fragment-based crystallographic screens have reported that $\mathrm{Br} 27$ and $\mathrm{Br} 6$ bind to the exo site and flap site respectively and stabilize the closed conformation of the flaps in the wild-type HIV-PR. ${ }^{[9]}$ However, to our knowledge the binding of these fragments to the $6 \mathrm{X}$ mutant has not been investigated yet. Moreover, flap flexibility is an inherently dynamic issue and thus, it cannot be entirely captured in a single crystal structure.

Analysis of the simulations reveals that $\mathrm{Br} 27$ is unable to maintain stable interactions with exo site residues and early detaches from this site during MD simulations. On the other hand Br6 remains bound to the flap site most of the time of the simulations, despite of the small size of Br6 and solvent exposure of flap site. Since only Br6 is able to maintain stable interactions within its native site as in the crystal structure, from now on the analysis is focused in this last fragment.

Figure 1 shows that when fragment Br6 is bound to flap site of 6X (i.e. system 6X/TL-3/ $\mathrm{Br6}$ ) the flaps remain closed along the entire MD simulations. That is, the binding of $\mathrm{Br} 6$ to the flap suppress the tendency to flap opening in the $6 \mathrm{X}$ mutant. Moreover, the closing of the flaps in 6X/TL-3/Br6 is even more stable than in Wt/TL-3 itself, as evidenced in Figure 1.

In replica 2 of complex $6 \mathrm{X} / \mathrm{TL}-3 / \mathrm{Br} 6$ the fragment detaches from the flap site during the simulations but it then re-attaches to another nearby site on the outside/top of the flap (Figure 2). Curiously, there seems to be no effect of the detachment of Br6 on the opening of 
the flaps (Figure 1). This is probably because concerted motion of flap residues would take longer than the time period of Br6 detachment/re-attachment cycle.

In view of these findings, the following question arises: How residues from the flap tip where Br6 is bound communicate with residues from the other flap tip to trigger the closing of the flaps? Is this communication established directly from flap to flap or it might be mediated by intermediate residues from other sites of the protein? In other words we want to know how the binding of $\mathrm{Br} 6$ to the flap site compensates the effect of the mutations and suppress the flap opening tendency in $6 \mathrm{X}$ mutant.

To attempt answering these questions, in the next section we performed a dynamical cross correlation analysis (DCCA).

\subsection{Dynamic Cross Correlation Analysis (DCCA)}

DCCA is a technique extensively applied for analysing communications among separate parts of a molecular system and is usually employed to characterize the correlation in motion of protein residues along MD trajectories. ${ }^{[21]}$ The correlation Cij between the residues falls in the range from -1 to 1 . If the residues move in the same direction in most of the frames, the motion is considered to be correlated, and $\mathrm{Cij}$ will be positive. If they move in opposite directions in most frames, the motion is said to be anti-correlated, and Cij will be negative. If the correlation value between the 2 residues is close to zero, then the motion is said to be uncorrelated. ${ }^{[21]}$

Figure 3 shows the Dynamic Cross Correlation Maps (DCCMs) of complexes Wt/TL-3, 6X/ TL-3 and 6X/TL-3/Br6. Also 3D visualization of the positive correlations is shown for each system.

An overall negative correlation can be observed between both flaps in the three systems (black circled zones in the DCCMs). However, in Wt/TL-3 and 6X/TL-3/Br6 there is also a positive correlation zone between the tips of the flaps as indicated by an arrow in the DCCMs and by the red lines connecting both flap tips on the 3D protein structure. The positive correlation means that both flap tips move in the same direction and so they are maintained closed together most of the time of the simulations, thus preventing inhibitor releasing. On the other hand in system 6X/TL-3 the positive correlation between flap tips is not observed which is in line with the fact that flaps move apart from each other along the simulations.

Further information can be extracted from the correlation matrix by diagonalizing it (i.e. solving the eigenvalue problem) and analysing the first eigenvectors or Principal Components (PCs) which capture the essential dynamic of protein systems. ${ }^{[13]}$

\subsection{Principal Component Analysis (PCA)}

Figure 4 shows the first principal component (PC1) for systems Wt/TL-3, 6X/TL-3 and 6X/ TL-3/Br6. Since Br6 is bound to chain A of the homo-dimer (right chain of figure 4, residues 1 to 98) below we discussed the PC1 changes on this chain on going from Wt/TL-3 to $6 \mathrm{X} / \mathrm{TL}-3$ to $6 \mathrm{X} / \mathrm{TL}-3 / \mathrm{Br} 6$. 
In the wild type complex the flap elbows experience the largest movements among the different segments of the protease, as indicated by length of the PC1 displacement vectors (Figure 4). Hence, it is fair to say that the dynamic of the entire protease in the wild type form is mostly driven by flap elbows dynamics. The displacement vectors show that flap elbow from one of the protease chains moves in a clockwise manner pushing backward the 80s loops which in turn push back the flap tip. At the same time the flap elbow from the other monomer moves in an anticlockwise manner so that both flap tips move together back and forth. These clockwise/anti-clockwise movements of the flap elbows involve some structural reorganization within each monomer. In particular, note that PC1 displacement vector from flap elbow point in different direction than PC1 vector from fulcrum suggesting that inter-residue interaction at the interface among these segments are affected due to flap elbow displacements.

On the other hand, in mutant variant bound to TL-3 (6X/TL-3) the clockwise/anti-clockwise movements of flap elbows is suppressed. Instead, flap elbow and fulcrum (as well as flap, cantilever and 80s loop from each monomer) move as a whole in an outward/inward manner, as indicated by the PC1 displacement vectors. As will be shown later, it seems that some of the point mutations on the protease reinforce the interactions at the interface between some of these segments preventing the clockwise/anticlockwise movement of flap elbows. As a consequence of the anti-correlated outward/inward movement of $6 \mathrm{X}$ protease monomers both flap tips get separated from each other. Curiously, when Br6 is bound to the flap site of 6X/TL-3 complexes, flap elbows behave much like in the wild type form, i.e. they both move in a clockwise/anticlockwise correlated manner so that flap tips stay close together.

It is worth noting that the relative displacements recovered by PC1 are in an overall agreement with DCCM maps of the three complexes (Figure 3). Thus, the PC1 alone captures at least qualitatively the essential dynamics of the protease in the different complexes.

In view of the previous results we would like to know in more detail how mutations on the protease change its dynamical behaviour. And more importantly, how is Br6 able to restore back the dynamic of the mutant variant into the dynamic of the wild type form of the protease?

To answer these questions the charge density topological analysis on extreme projections along PC1 was performed next.

\subsection{Charge Density Topological Analysis}

Figures 5, 6 and 7 show the extreme projections along PC1 for the Wt/TL-3, 6X/TL-3 and $6 \mathrm{X} / \mathrm{TL}-3 / \mathrm{Br} 6$ complexes, respectively. Topological elements of the charge density were computed on selected zones of the extreme structures to reveal key interactions among structural segments of the protease.

Graphical representation of charge density topological elements (i.e. molecular graphs) allows capturing weak, hydrophobic interactions among different HIV-PR segments. These 
hydrophobic interactions govern the protein dynamics and would be missed in a merely geometric analysis of the interactions.

Moreover, the charge density values at the interactions Bond Critical Points (BCPs) are considered as a measure of the strength of those interactions which allow us to measure whether the connections between any two segments of the HIV-PR become strengthened or otherwise weakened upon residue mutations or fragment binding.

Figure 8 shows the charge density networks between segments of the protease for the three complex systems studied here.

Expanded zones in the upper part of Figures 5, 6 and 7 show the interaction network involving both protease flap tips. In complexes Wt/TL-3 and 6X/TL-3/Br6 there are bond paths connecting backbone atoms of both flap tips to one another in both $\mathrm{PC} 1$ extreme projection structures which are in line with a correlated movement of flap tips.

While in complex 6X/TL-3, because of the anti-correlated motion of flaps tips, they are close enough for direct interaction in only one of the extreme projections. Therefore, bond paths connecting both flap tips backbone atoms are only observed in maximum PC1 extreme projection structure. Moreover, the charge density networks in Figure 8 show that interactions between both flap tips are stronger in the first complexes than in last one which is also in line with the fact that flap tips stay close together in the first case but open apart in the last one.

More clues about origin of different behaviour of flap tips can be found by inspecting flap elbow interactions. Expanded zones to the right of Figures 5, 6 and 7 show the main interactions of flap elbow with neighbouring segments in monomer A of protease complexes.

As indicated by arrows in Figure 5 (right panel) for the wild type complex, on going from the minimum PC1 projection (purple) to the maximum one (cyan) the flap elbow moves in anti-clockwise manner bringing forward the 80s loop which in turn causes the forward shift of flap tip. The displacement of flap elbow causes the rupture of hydrogen bonds (H-bonds) that Asn37 and Met36 from this segment form with fulcrum residues Gln18 and Lys20, respectively and also cause the rupture of interaction between Asn37 with cantilever residue Tyr59. Thus, transition from minimum to maximum PC1 projection structures involves the breaking of (at least) three H-bonds that hold flap elbow in place. At the same time, in flap elbow from the other protease monomer the inverse changes are occurring so that enthalpy penalty of breaking $\mathrm{H}$-bonds in one monomer might be compensated by their formation in the other.

On the other hand, in the mutant variant 6X/TL-3 the flap elbow moves outward on going from minimum to maximum $\mathrm{PC} 1$ projection, as indicated by the arrows in Figure 6, right expanded zone.

Note in this figure that cantilever residue Pro63, which is one of the mutated residues in 6X variant, is connected through bond paths to residue Gly 16 from fulcrum tip, in both extreme 
projections. While in the wild type complex residue Leu63 does not form any strong interaction with fulcrum tip as depicted in Figure 5, right panel.

In complex 6X/TL-3, the O@P63・•••H@G16 interaction holds the fulcrum tip close to flap elbow in such a way that they cannot be detached from each other. Note that, unlike the wild type complex, the interactions of elbow with fulcrum residues Gln18 and Lys20 are still formed upon transition from one extreme structure to the other. Therefore, as flap elbow moves in outward manner, it drags with it the fulcrum tip and cantilever so that the three segments move together as a whole.

As indicated by the arrows in Figure 6 (right expanded zone) this mass movement involves a downward shift of cantilever and outward/downward movement of the contiguous flap segment, which explains at least in part the PC1 displacement vectors of complex 6X/TL-3 in Figure 4.

Moreover, another point mutation V66I also seems to be involved in the outward movement of flaps in mutant complex 6X/TL-3. In this complex, residue Ile77 at 80s loop extends down the flap elbow and forms interactions with side chain of Met36 which in turn is connected through bond paths to Lys20 at fulcrum (Figure 6, right panel). Thus, Ile77 is connected via Met36 with fulcrum resides and so, the motion of 80s loop is correlated with movement of fulcrum, elbow and cantilever, as indicated by the orange arrows in Figure 6, right panel. In turn, 80s loops drags down with it flap base residues since both segments are forming a $\beta$-sheet motif and so they are firmly associated to each other. On the other hand, in wild type complex the shortest side chain of Val77 cannot reach Met36 side chain and so its motion is not correlated with that of fulcrum segment (note in Figure 5 that there isn't any topological element connecting Val77 with Met36 in the wild type complex).

Thus, these point mutations (i.e L63P and V77I) seem to increase the strength of the interactions among different segments of the protease thus preventing the clockwise/anticlockwise movement of flap elbows necessary for a correlated motion of both flaps tips. As evidenced from the charge density networks of Figure 8, the edges connecting most of the segments of the protease are quite wider in complex 6X/TL-3 than in wild-type complex, which further supports the previous statement.

Regarding ternary complex $6 \mathrm{X} / \mathrm{TL}-3 / \mathrm{Br} 6$ its protein backbone shift is quite more subtle than in previous systems, on going from one extreme projection to the other (see Figure 7, right expanded zone). The most dramatic shift occurs in the flap elbow which, as in wild type complex, experiences an anti-clockwise movement on going from minimum to maximum $\mathrm{PC} 1$ projection structure. It is worth noting the rupture of the strong salt bridge between Arg57 at flap base and Glu35 at elbow that is otherwise formed in PC1 extreme projection structures of complexes 6X/TL-3 and Wt/TL-3. By inspecting Figure 7 it is evident that Br6 directly perturbs the salt bridge as evidenced by the bond paths connecting the Br6 bulky bromine atom with R57 side chain atoms. This strong interaction (i.e. R57 to E36) holds firmly the flap elbow to the flap base, hence it is expected that after its disruption flap elbow could move more freely and independently from other segments of the protease thus mimicking the wild type elbow motion. In fact note in the charge density network of ternary 
complex 6X/TL-3/Br6 that interactions between flap and elbow becomes much weaker after Br6 has bound to $6 \mathrm{X} / \mathrm{TL}-3$ (Figure 8 ). Also interactions of elbow with fulcrum and 80s loop residues become weaker after fragment bound to flap site. Thus, the result of salt bridge disruption is the release of flap elbow in the resistant form of the protease.

\section{Conclusions}

In this work we have performed long MD simulations of wild type and 6X mutant form of HIV-PR bound to potent inhibitor TL-3. The simulations show that on going from Wt/TL-3 to $6 \mathrm{X} / \mathrm{TL}-3$ the equilibrium shifts from closed to semi-open conformation of the flaps, in agreement with previous experimental and theoretical evidence. Then we placed small fragments Br6 and Br27 to the flap and exo sites of 6X/TL-3 and performed MD simulations of the ternary complexes $6 \mathrm{X} / \mathrm{TL}-3 / \mathrm{Br} 6$ and $6 \mathrm{X} / \mathrm{TL}-3 / \mathrm{Br} 27$. Surprisingly one of the fragments, Br6, was able to restore back the enzyme to closed conformation of the flaps.

PCA and QTAIM charge density analysis provided some clues about the mechanism of flap opening and closing in 6X/TL-3 and 6X/TL-3/Br6, respectively. Mutations L63P and V77I seem to increase the strength of the interactions among different segments of $6 \mathrm{X} / \mathrm{TL}-3$ thus preventing the clockwise/anti-clockwise movement of flap elbows required for keeping both flap tips closed together in a correlated motion. On the other hand when Br6 is placed at flap site of $6 \mathrm{X} / \mathrm{TL}-3$, it perturbs the R57•••E35 salt bridge that hold firmly the flap elbow to flap base. Thus, the result of salt bridge disruption is the release of flap elbow and restoration of clockwise/anti-clockwise motion in the resistant form of the protease. In other words, small fragment $\mathrm{Br} 6$ once bound to flap site of $6 \mathrm{X}$ protease variant is able to turn the dynamics of mutant protease variant into the dynamics of the wild type form. It still remains to be proven if these results are reproducible in vitro. If that were the case, small fragments could be added to AIDS patient's treatment plans to overcome resistance to HIV-PR inhibitors.

\section{Acknowledgements}

We acknowledge SECyT-UNNE and CONICET and NIH (Grant R01 GMO69832, S.F. and A.J.O.) for financial support. The authors also acknowledge the use of CPUs from the High Performance Computing Center of the Northeastern of Argentina (CECONEA). The Geforce GTX Titan X GPU used for this research was donated by the NVIDIA CorporationReferences.

\section{References}

[1]. Goodsell DS; Protein Data Bank 2000 doi:10.2210/rcsb_pdb/mom_2000_6

[2]. Yu Y, Wang J, Shao Q, Shi J, Zhu W; Sci. Rep 2015, 5, 10517. [PubMed: 26012849]

[3]. Perryman AL, Lin J; Prot. Sci 2004, 13,1108-1123.

[4]. Heaslet H, Kutilek V, Morris GM, Lin Y, Elder JH, Torbett BE, Stout CD, J. Mol. Biol 2006, 356, 967-981. [PubMed: 16403521]

[5]. Ali A, Bandaranayake RM, Cai Y, King NM, Kolli M, Mittal S, Murzycki JF, Nalam MNL, Nalivaika EA, Özen A, Prabu-jeyabalan MM, Thayer K, Schiffer CA, Viruses 2010, 2, 2509_ 2535. [PubMed: 21994628]

[6]. Ung PMU, Dunbar JB, Gestwicki JE, Carlson HA. J. Med. Chem 2014, 57, 6468-6478. [PubMed: 25062388]

[7]. Perryman AL, Zhang Q, Soutter HH, Rosenfeld R, McRee DE, Olson AJ, Elder JE, Stout CD, Chem. Biol. Drug. Des, 2010, 75, 257-268. [PubMed: 20659109] 
[8]. Tiefenbrunn T, Forli S, Baksh MM, Chang MW, Happer M, Lin Y, Perryman AL, Rhee J, Torbett BE, Olson AJ, Elder JH, Finn MG, Stout CD, ACS Chem. Biol 2013, 8, 1223-1231. [PubMed: 23540839]

[9]. Tiefenbrunn T, Forli S, Happer M, Gonzalez A, Tsai Y, Soltis M, Elder JH, Olson AJ, Stout CD, Chem. Biol. Drug. Des 2014, 83, 141-148. [PubMed: 23998903]

[10]. a)Case DA, Cheatham TE, Darden T, Gohlke H, Luo R, Merz KM Jr., Onufriev A, Simmerling C, Wang B, Woods RJ, J. Comput. Chem 2005, 26, 1668-1688; [PubMed: 16200636] b)Maier JA, Martinez C, Kasavajhala K, Wickstrom L, Hauser KE, Simmerling C, Chem. Theory Comput 2015, 11, 3696-3713;c)Wang J, Wolf RM, Caldwell JW, Kollman PA, Case CA, J Comput Chem 25, 2004,1157-1174. [PubMed: 15116359]

[11]. Grant BJ, Rodrigues APC, ElSawy KM, McCammon JA, Caves LSD, Bioinformatics 2016, 22 , 2695-2696.

[12]. Bakan A, Meireles LM, Bahar I, Bioinformatics 2011, 27, 1575-1577. [PubMed: 21471012]

[13]. Skjaerven L, Martinez A, Reuter N, Prot. Struct. Func. Bioinf 2011, 79, 232-243.

[14]. Bader RFW, Atoms in Molecules: A Quantum Theory. Oxford University Press, New York, 1990.

[15]. Gutierrez LJ, Angelina EL, Gyebrovszki A, Fülöp L, Peruchena NM, Baldoni HA, Penke B, Enriz RD, J. Biomol. Struct. Dyn 2016, 35, 413-426. [PubMed: 26813690]

[16]. Luchi AM, Angelina EL, Andujar SA, Enriz RD, Peruchena NM, J. Phys. Org. Chem 2016, 29, 645-655.

[17]. Andujar SA, Tosso RD, Suvire FD, Angelina E, Peruchena N, Cabedo N, Cortes D, Enriz RD, J. Chem. Inf. Model 2012, 52, 99-112. [PubMed: 22146008]

[18]. Tosso RD, Andujar SA, Gutierrez L, Angelina E, Rodríguez R, Nogueras M, Baldoni H, Suvire FD, Cobo J, Enriz RD, J. Chem. Inf. Model 2013, 53, 2018-2032. [PubMed: 23834278]

[19]. Frisch MJ, Trucks GW, Schlegel HB, Scuseria GE, Robb MA, Cheeseman JR, Scalmani G, Barone V, Petersson GA, Nakatsuji H, Li X, Caricato M, Marenich A, Bloino J, Janesko BG, Gomperts R, Mennucci B, Hratchian HP, Ortiz JV, Izmaylov AF, Sonnenberg JL, WilliamsYoung D, Ding F, Lipparini F, Egidi F, Goings J, Peng B, Petrone A, Henderson T, Ranasinghe D, Zakrzewski VG, Gao J, Rega N, Zheng G, Liang W, Hada M, Ehara M, Toyota K, Fukuda R, Hasegawa J, Ishida M, Nakajima T, Honda Y, Kitao O, Nakai H, Vreven T, Throssell K, Montgomery JA Jr., Peralta JE, Ogliaro F, Bearpark M, Heyd JJ, Brothers E, Kudin KN, Staroverov VN, Keith T, Kobayashi R, Normand J, Raghavachari K, Rendell A, Burant JC, Iyengar SS, Tomasi J, Cossi M, Millam JM, Klene M, Adamo C, Cammi R, Ochterski JW, Martin RL, Morokuma K, Farkas O, Foresman JB, and Fox DJ, Gaussian 09, Revision D.01. Gaussian, Inc., Wallingford, 2016.

[20]. Lu T, Chen F, J. Comput. Chem 2012, 33, 580-592. [PubMed: 22162017]

[21]. Sethi A, Eargle J, Black A, Luthey-Schulten Z, Proc. Natl. Acad. Sci. U. S. A 2009, 106, 66206625. [PubMed: 19351898] 


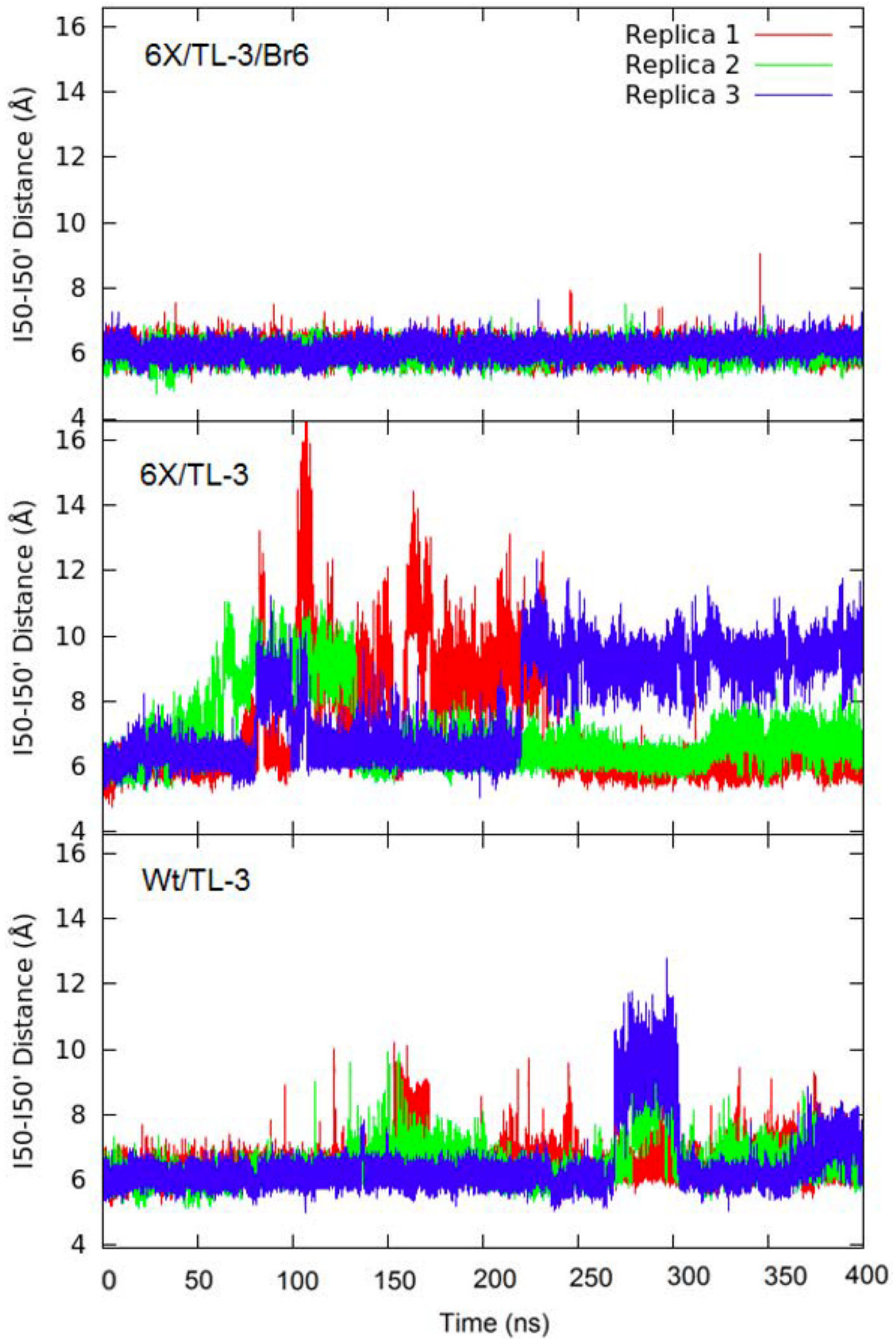

Figure 1.

Backbone distance Ca@I50‥Ca@I50’ between both flap tips along MD trajectories. 

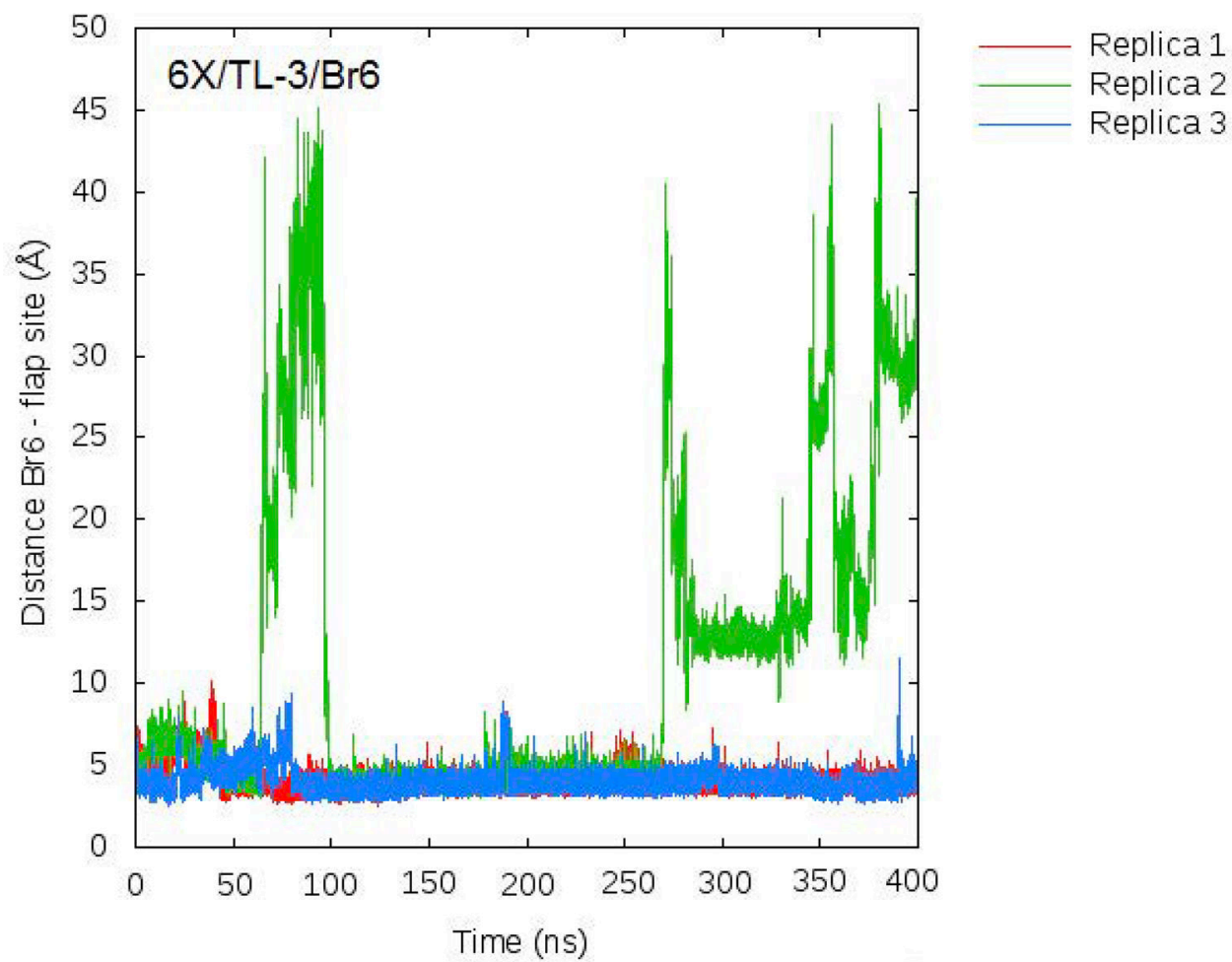

\section{Replica 3}

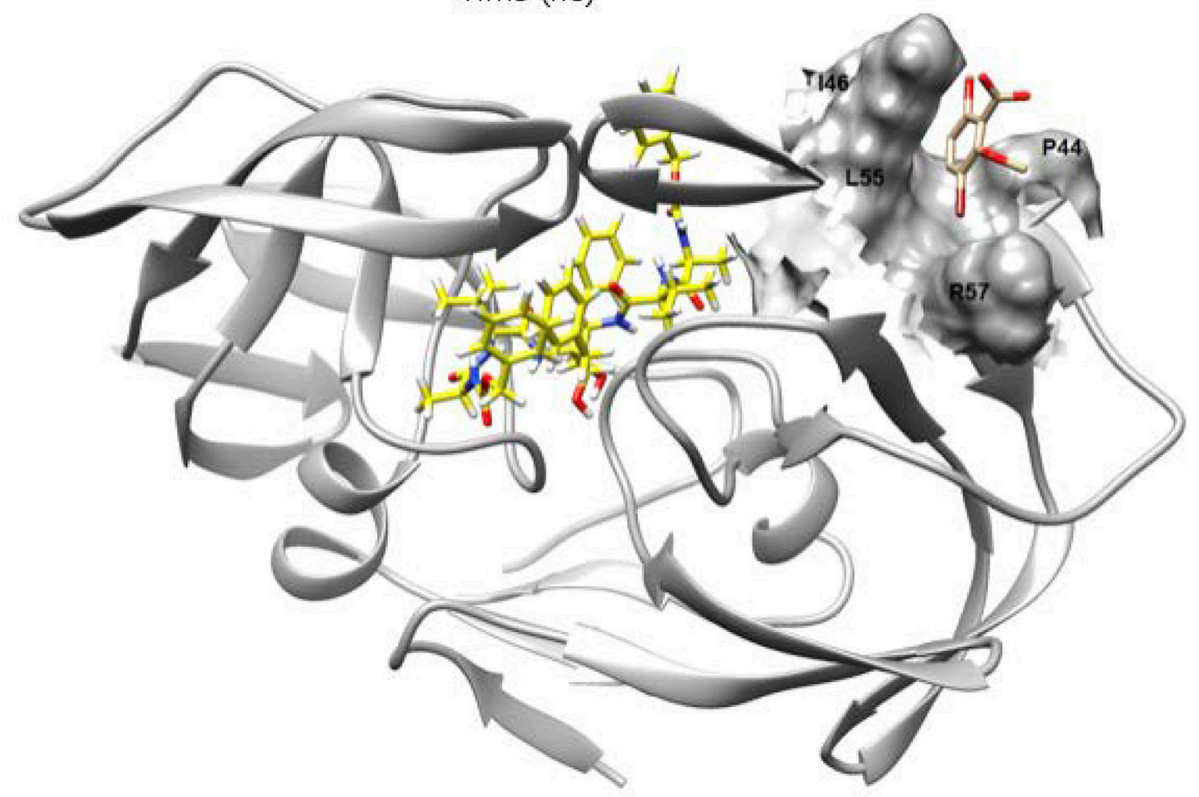

Figure 2.

Distance between center of mass (CM) of small fragment Br6 and CM of flap site along MD trajectories of complex 6X/TL-3/Br6. Flap site is defined by residues L55, R57, I46 and P44 that conform the walls of Br6 binding pocket in $6 \mathrm{X}$ mutant. A snapshot of Br6 binding pocket in $6 \mathrm{X}$ protease variant is shown at the bottom of figure. 

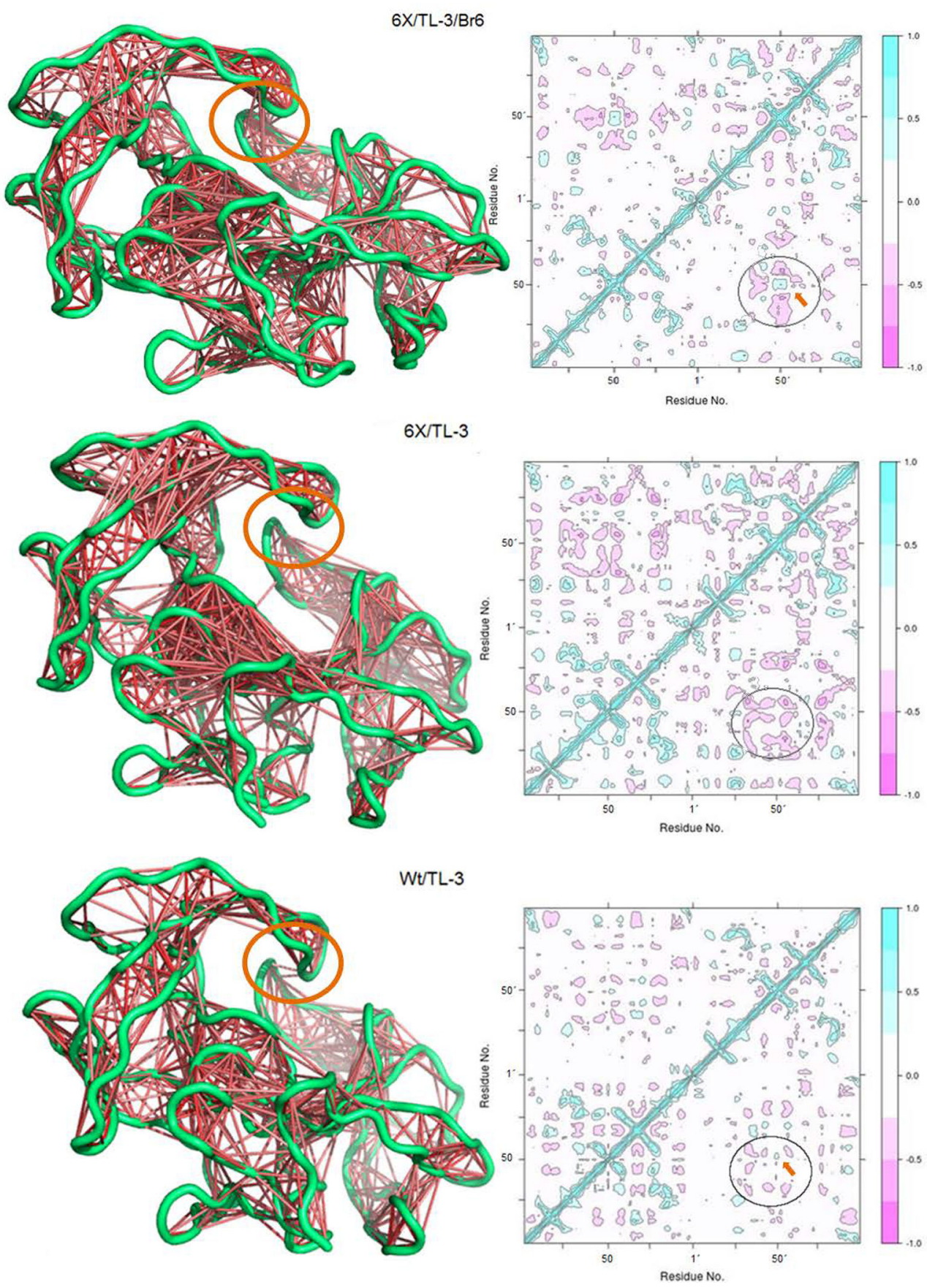

Figure 3.

Correlations between protease residues in complexes Wt/TL-3, 6X/TL-3 and 6X/TL-3/Br6. On the right, the two-dimensional Dynamic Cross Correlation Maps (DCCMs) are depicted. On the left, the positive correlations between residues were drawn as red lines on the protein structure. Note that while flaps are mostly negatively correlated (circled zone in the DCCMs) there is a positive correlation region between flaps tips in complexes Wt/TL-3 and 6X/TL-3/Br6 (indicated with an arrow in the DCCMs and by the red lines connecting both flaps on the 3D protein structure) 


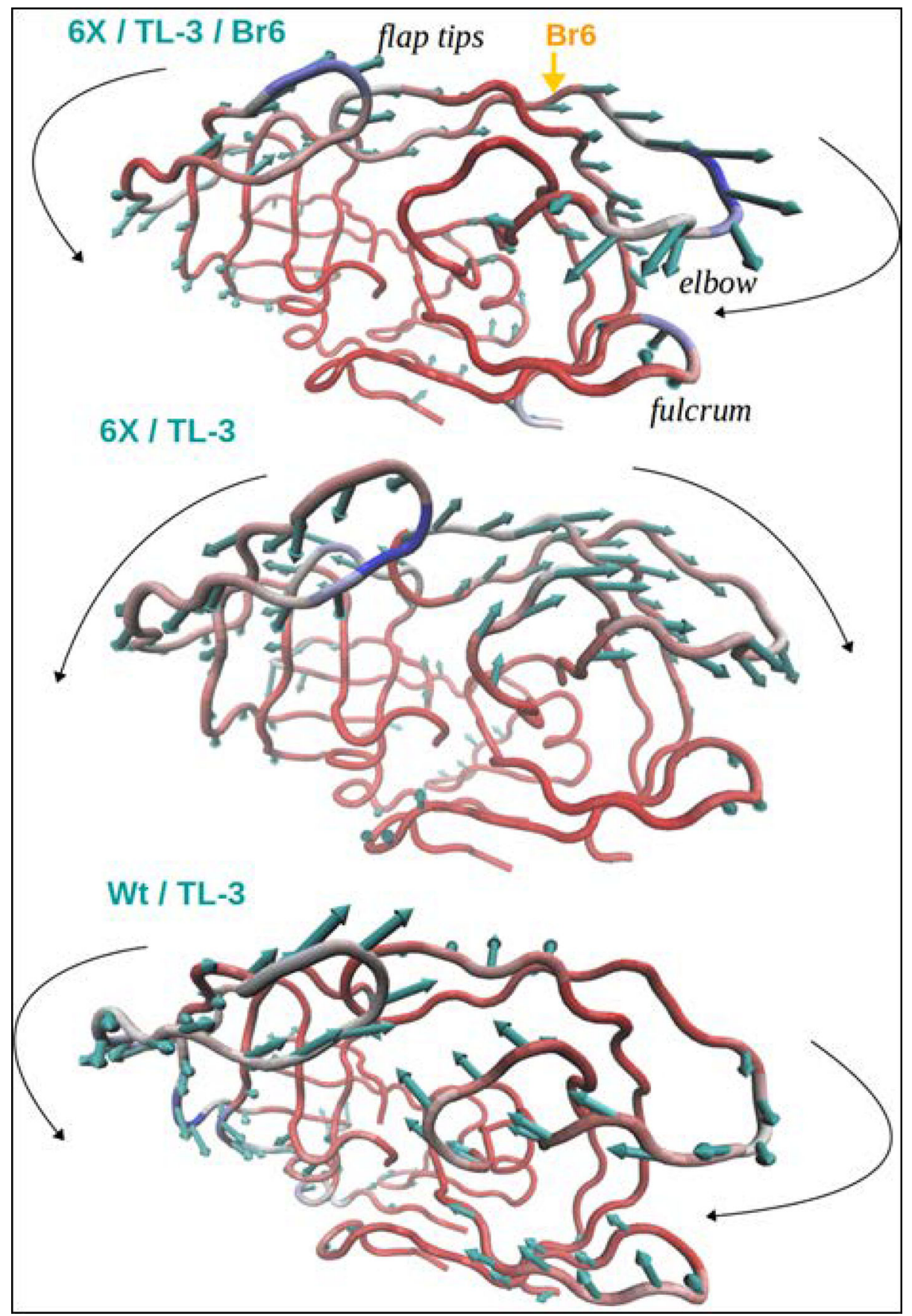

Figure 4.

Vector field representation of PC1 obtained from the MD trajectories. 


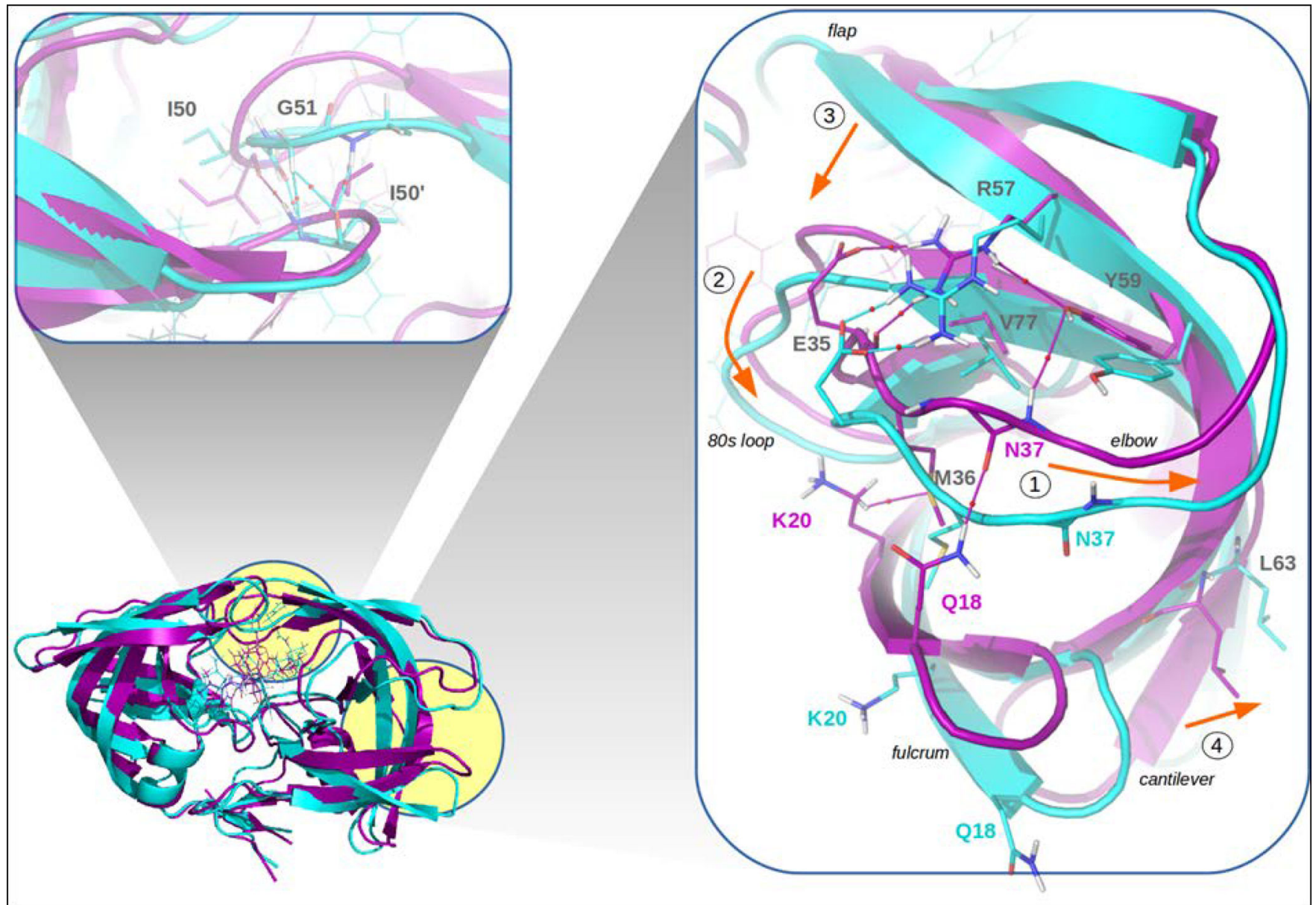

Figure 5.

Extreme projections along PC1 for the Wt/TL-3 complex (maximum and minimum PC1 projections are depicted in cyan and purple, respectively). Topological elements of the charge density are shown for selected zones on the extreme structures. Bond paths connecting the nuclei are depicted in cyan and purple for maximum and minimum projections, respectively and the small red circles on them show the bond critical points (BCPs). 


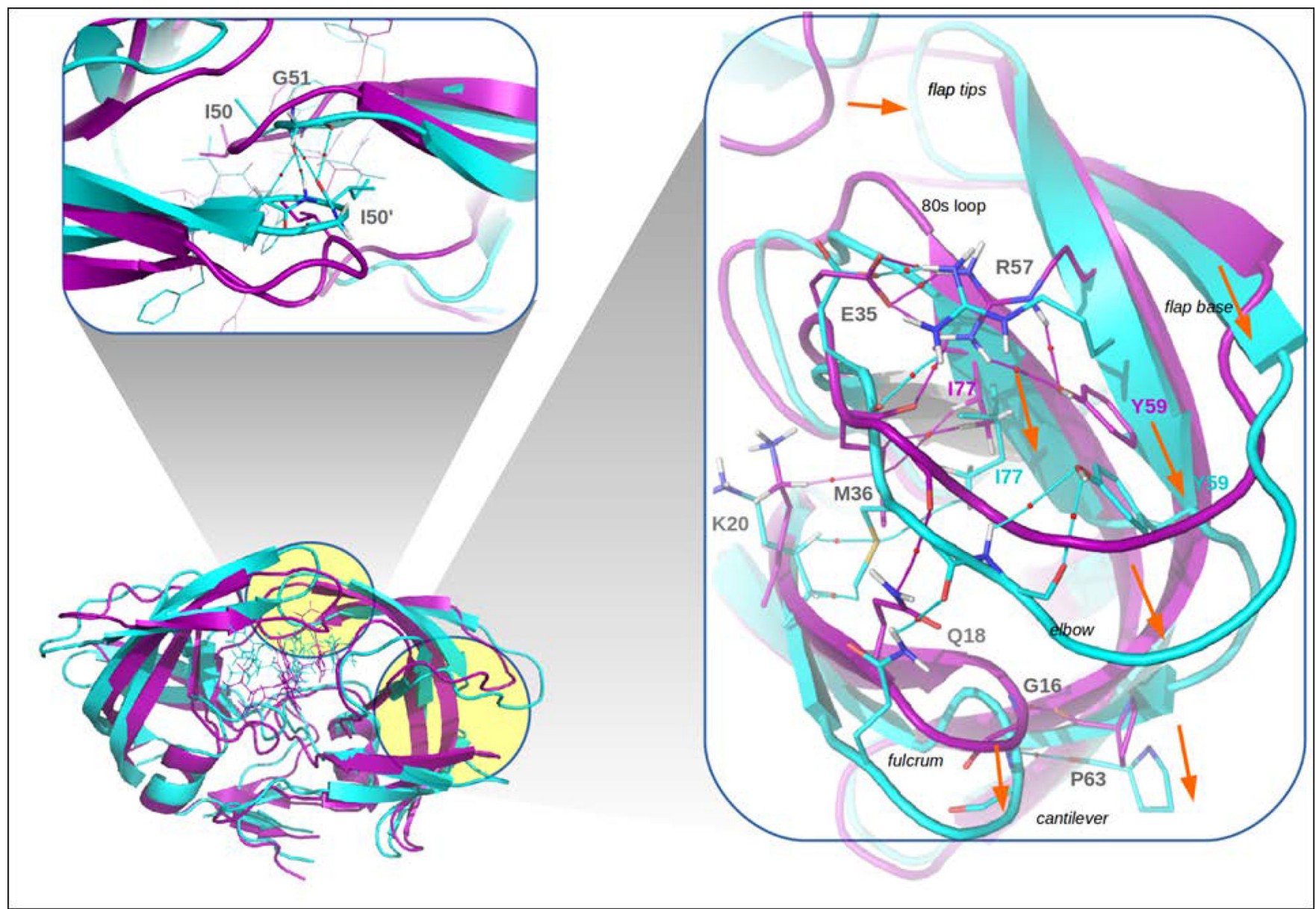

Figure 6.

Extreme projections along PC1 for the mutant $6 \mathrm{X} / \mathrm{TL}-3$ complex (maximum and minimum PC1 projections are depicted in cyan and purple, respectively). Topological elements of the charge density are shown for selected zones on the extreme structures. Bond paths connecting the nuclei are depicted in cyan and purple for maximum and minimum projections, respectively and the small red circles on them show the bond critical points (BCPs). 


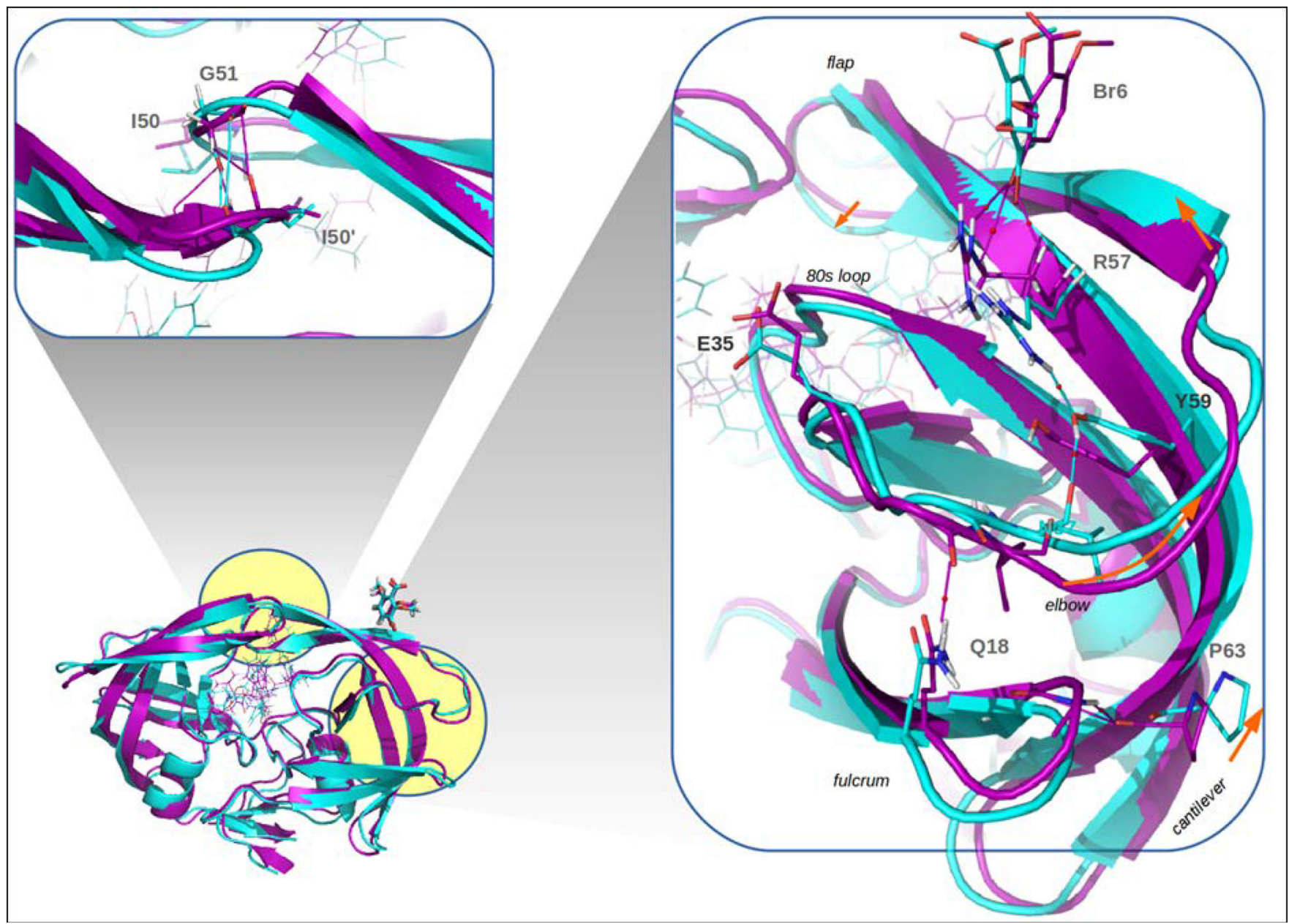

Figure 7.

Extreme projections along PC1 for the mutant 6X/TL-3/Br6 complex (maximum and minimum PC1 projections are depicted in cyan and purple, respectively). Topological elements of the charge density are shown for selected zones on the extreme structures. Bond paths connecting the nuclei are depicted in cyan and purple for maximum and minimum projections, respectively and the small red circles on them show the bond critical points (BCPs). 


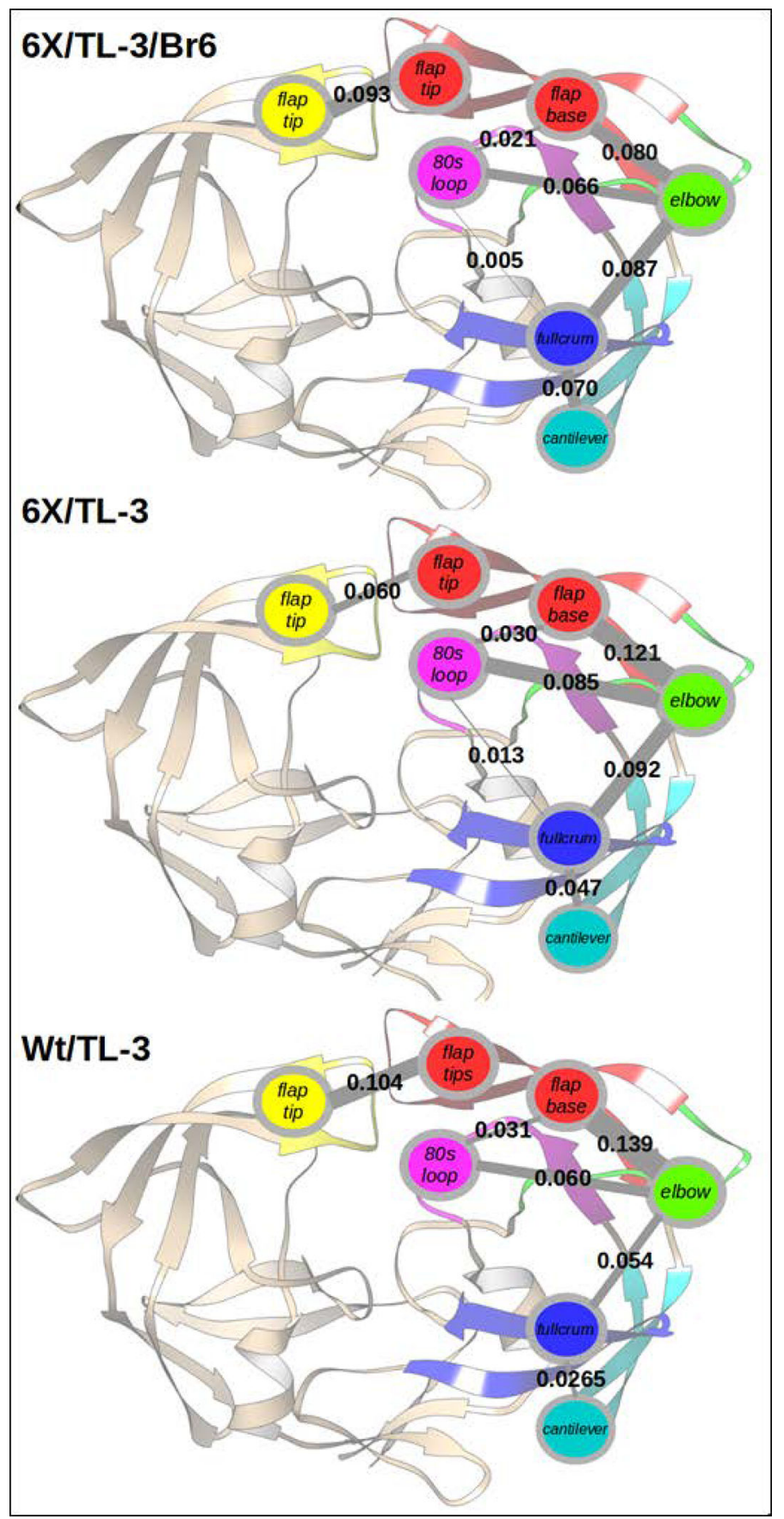

Figure 8.

Charge density networks for the three complex systems studied here. Each node of the network represents a community of residues from a particular segment of the protease and the width of edges connecting the nodes are proportional to the sum of charge density values at the inter-segment bond critical points, averaged over the two PC1 extreme projection structures $\left(\sum \rho_{b}\right)$. The $\Sigma \rho_{b}$ values (in atomic units) are depicted on the corresponding edges. 